\title{
Over-expression of $\beta$-carotein hydroxylase (chyB) gene affects green and albino leaves ultrastructure in Arabidopsis thaliana
}

\author{
Tchouopou Lontchi Josine ${ }^{1}$, Jing Ji ${ }^{1 ⿴}$, Gang Wang1, Qing Zhao ${ }^{2}$
}

\begin{abstract}
Albinism is a common problem encountering breeding and interspecific crosses. Arabidopsis thaliana chyB (AtchyB) gene encodes for $\beta$-carotene hydroxylase. We generated transgenic plant over-expressing AtchyB in A. thaliana and compared to wild-type (WT) plants in response to light stress. We tested whether the over-expression of $A t c h y B$ gene can affect the green and albino leaf ultrastructure in $A$. thaliana transgenic lines. In this study, over-expression of AtchyB cDNA governed by Cauliflower Mosaic_Virus_35S (CaMV 35S) promoter showed a progressive yellow coloration in primary leaves and ultrastructural changes to albino and green leaves of transgenic $A$. thaliana plant in comparison to wild type (WT). Leaf albinism causes dilatation of both the palisade parenchyma and the secretory cells, enlargement of plastoglobules and increased cuticle thickness. In addition, the presence of low number of chloroplasts and/or starch grains is associated with leaf albinism phenomenon. Altogether, this suggests that $A$. thaliana 35S:AtchyB transgenic lines induce leaf
\end{abstract}

Received: 28 September 2014

Accepted revised version: 24 November 2014

Published online: 16 January 2015

(C) Josine et al (2015)

Publisher: Horizon e-Publishing Group

CITATION

Josine, T. L. , J. Ji, G. Wang, and Q. Zhao. 2015. Over-expression of $\beta$-carotein hydroxylase (chyB) gene affects green and albino leaves ultrastructure in Arabidopsis thaliana. Plant Science Today 2(1): 29-37. doi:10.14719/pst.2015.2.1.85

AUTHOR'S AFFILIATIONS

${ }^{1}$ School of Environmental Science \& Engineering, Institute of genetic Engineering,Tianjin University, Tianjin 300072, P. R. China

2 School of Chemical Engineering \& Technology, Tianjin University, Tianjin 300072, P. R. China

CORRESPONDENCE

\Prof. Jing Ji - E-mail jijingtjdx@163.com; Tel: +86-22-87402200 albinism which affects the leaves ultrastructure with retrograde signaling, defects in chlorophyll biosynthesis and chloroplast dysfunction.

Keywords: transgenic; AtchyB; light stress; phenomenon; chloroplast

Abbreviations: CTAB; cetyltrimethyl ammonium bromide; YEP; Yeast Extract and Peptone; TEM; Transmission Electron Microscope; DEPC; diethyl pyrocarbonate; cDNA; complementary Deoxyribonucleic acid; RT-PCR; real time polymerase chain reaction

\section{Introduction}

Breeding and interspecific crosses in plants commonly cause albinism. It is characterized by partial or complete loss of chlorophyll pigments and incomplete differentiation of chloroplast membranes. Genetic factors are even more important in determining the frequency of albino plant formation, and are major determinants in albinism. Albino leaf tissues are found in many different plant species because of lack of chlorophyll pigments (Abadie et al., 2006; Maya et al., 2009).

Arabidopsis thaliana (A. thaliana) has been intensively used as a model plant for transgenic studies of physiological, biochemical and ultrastructural processes. A. thaliana over-expressing the chy $B$ gene that encodes for beta-carotene hydroxylase were found more tolerant to conditions of high light and high temperature, with a reduced leaf necrosis, reduced production of the stress indicator anthocyanin and reduced lipid peroxidation, due to the function of zeaxanthin in preventing oxidative damage of membranes (Davison et al., 2002). A. thaliana early chloroplast biogenesis 1 (AtECB1), an allele of mesophyll-cells RNAi library line 7 (MRL7), was identified due to the regulation of plastid-encoded RNA polymerase (PEP) function and chloroplast biogenesis (Yua et al., 2014). The knockout lines for AtECB1 displayed albino phenotype and impaired chloroplast 
development. In rice bran, Postharvest yellowing (PhY) was detected with an active metabolic response to high temperature and moisture stress due to the increase in total carotenoids that causesd yellowing in endosperm during conditions of high temperature (Helen and Eric, 2014). Etioplast and plastoglobules have been shown to be involved in plant stress response. Their number and size vary during plastid development and differentiation, and strongly increase during salt or light stress (Abdelkader et al., 2007; Claire et al., 2007).

pds gene silencing in Nicotiana tabacum plants showed different degrees of albinism, The grana stacking of thylakoid in variegation part was as normal as in the control. Etioplast occurred in the white sector of leaf (Min et al., 2009). AtchyB gene was identified to induce physiological metabolic activity, nutritional ability, high total carotenoids content and leaf yellowing in AtchyB transgenic A. thaliana plants (Tchouopou et al., 2013). The goal of the current work was to study at the ultrastructural level, how the over-expression of AtchyB gene to A. thaliana can affect energy absorption and photosynthesis of yellow and green leaf pigmentations.

\section{Material and Methods}

\section{Strain and plasmid}

The total RNA of $A$. thaliana was extracted with TRIzol ${ }^{\circledR}$ reagent (Invitrogen) and treated with DNase free of RNase. $2 \mu \mathrm{g}$ of total RNAs were used to synthesize first strand cDNA using the RevertAidTM
First Strand cDNA Synthesis Kit (Fermentas). The AtchyB gene was (NM_001036638.2) driven by the 35S cauliflower mosaic virus (CaMV35S) promoter; the NPT II was flanked by CaMV35S promoter and terminator. The plasmid pCambia2300-AtchyB was constructed as described Weidang et al. (2012). Agrobacterium tumefaciens strain C58 was used as the T-DNA donor.

\section{Agrobacterium tumefaciens mediated transformation method}

The wild type (WT) A. thaliana ecotype "Columbia" was used for Agrobacterium tumefaciens (C58 harbouring the plasmid pCambia2300-AtchyB) mediated transformation using the floral dip transformation method as previously described by Qi et al. (2004). The A. tumefaciens C58 cells carrying the binary vectors were grown in YEP $(1 \%$ Yeast Extract and $2 \%$ Peptone) liquid medium supplemented with $100 \mathrm{mg} / \mathrm{l}$ Kanamycin. WT $A$. thaliana seeds were previously soak in $1.5 \mathrm{ml}$ water containing ependorf for two days, then one or two seeds were planted per pot of mixture Vermiculite and Perlite (1:1) for germination. Plants were grown in a growth chamber with $50-70 \%$ relative humidity and a photoperiod of $16 \mathrm{~h}$ under natural day/night conditions and temperature of $22 / 18{ }^{\circ} \mathrm{C}$ respectively. Floral dip transformation started at the initial flowering stage and once every week over 3weeks, and then cultured to mature seeds. Dry T1 seeds were collected and kept at $4^{\circ} \mathrm{C}$ until use. They were selected on $1 / 2 \mathrm{MS}$ medium

Table 1. Primers used in amplification analysis of AtchyB, CaMV 35 sromoter, NPT II and Actin coding sequences.

\begin{tabular}{lll}
\hline Gene fragment & Forward & Reverse \\
\hline AtchyB & 5'- TCAACCGCCGTTACATTC-3' & 5' - AGAAGCGTGCCACAGAGC-3' \\
CaMV 35S promoter & 5'-AACAGAACTCGCCGTAAAG-3' & 5'-TAGTGGGATTGTGCGTCAT-3' \\
NPT II & 5'-CGATACCGTAAAGCAGAGGAA-3' & 5'-CACTGAAGCGGGAAGGGACT-3' \\
\hline
\end{tabular}
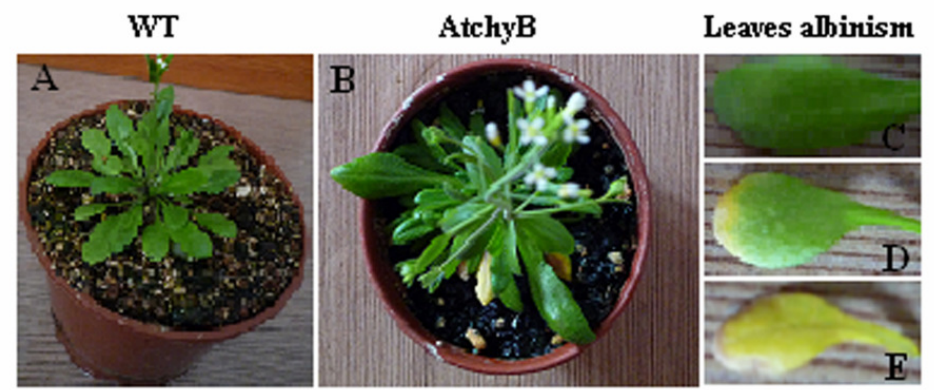

Fig. 1 Phenotypic variation between four weeks old WT and $A$. thaliana over-expressing the AtchyB gene (T1 plant). A: WT plant; B: Transgenic A. thaliana plant over-expressing the AtchyB gene, presence of yellow primary leaves; C, D, E: leaf albinism evolution from green to yellow. 
A

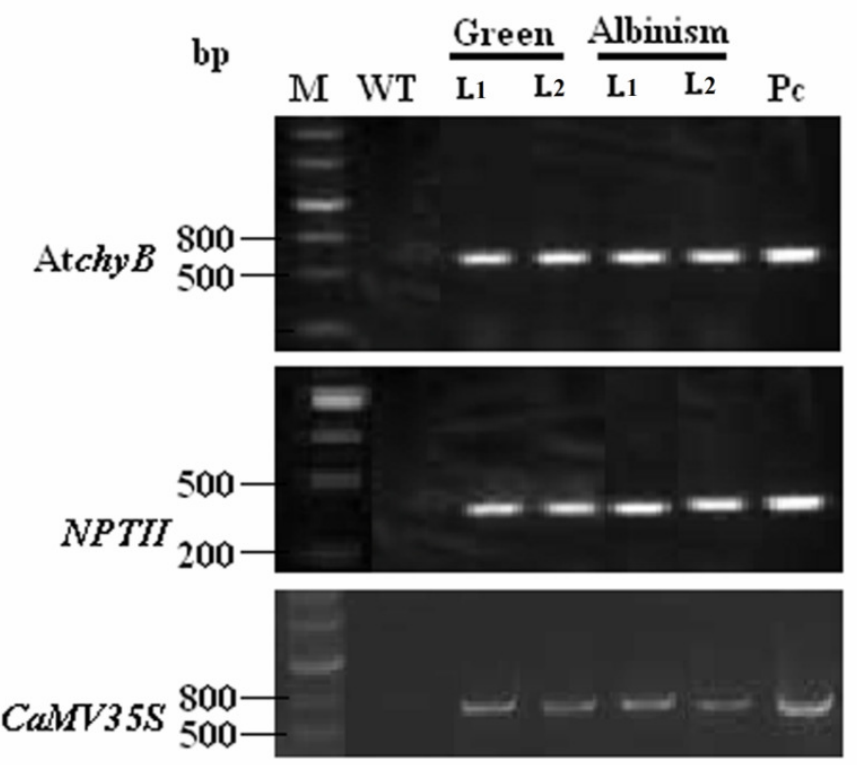

B

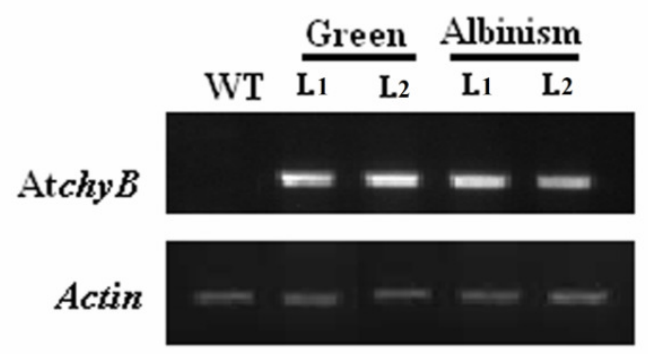

Fig. 2 PCR and RT-PCR amplification of kanamycin-tolerant plants. (A) AtchyB, CaMV 35S and NPT II PCR amplification analysis of kanamycin resistant lines 1 and 2 for their green and albino leaves. (B) RT-PCR amplification analysis of $A t c h y B$ gene expression in lines 1 and 2 green and albinism leaves. Cellular actin was used as internal control. Lane M is Marker III; lane WT is a negative control; lanes 1 and 2 are the two kanamycin tolerant lines over-expressing the AtchyB gene; lane Pc is a positive control.

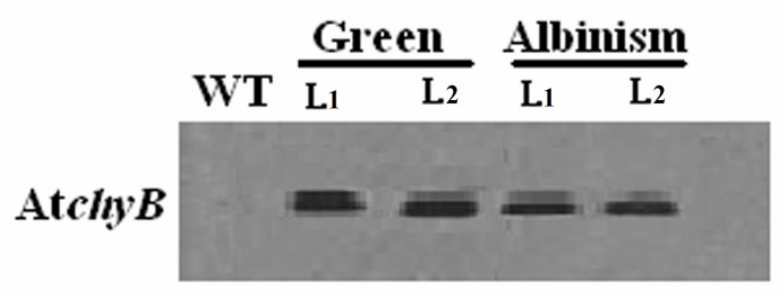

Fig. 3 Southern blotting analysis of the DNA in transgenic lines 1 and 2. WT: Wild Type of A. thaliana; L1 and L2: two lines of the $\mathrm{T}_{1} A$. thaliana Atchy $B$ transgenics. Digested DNA separated electrophoretically on a $1.0 \%$ (w/v) agarose gel and transferred onto Hybond N+ blotting membrane (Roche Diagnostics). Probes were produced using DIG labeled a 477 bp AtchyB gene fragment as a probe by PCR DIG Probe Synthesis (Roche Diagnostics).

supplemented with $100 \mathrm{mg} / \mathrm{L}$ Kanamycin and cultivated for 5 weeks. Kanamycin resistant plants were potted and grew to mature seeds. Identified transgenic lines through AtchyB gene amplification,
RT-PCR and northern blot were confirmed by analyzing southern blotting for the AtchyB gene expression in non transgenic and selected two transgenic lines (L1 and L2) of the $\mathrm{T}_{1}$ generation. 


\section{Total RNA isolation and cDNA synthesis}

Total RNA of $A$. thaliana green and albino leaves was extracted with TRIzol ${ }^{\circledR}$ reagent (Tiangen). All eppendorf tubes used for RNA isolation and RT-PCR were treated in the $0.1 \%(\mathrm{v} / \mathrm{v})$ diethyl pyrocarbonate (DEPC) water for $24 \mathrm{~h}$. Sampled $100 \mathrm{mg}$ of AtchyB transformants lines and WT leaf tissue frozen in liquid nitrogen and kept in $-70^{\circ} \mathrm{C}$ fridge until use; total RNA was then extracted according to TRIzol ${ }^{\circledR}$ plus RNA purification kit manuals (Invitrogen, USA). After treatment with RNase-free DNase I (Invitrogen) at $37^{\circ} \mathrm{C}$ for $30 \mathrm{~min}$ to remove genomic DNA contamination, the first strand of cDNA was synthesized using the RevertAid $^{\mathrm{TM}}$ First Strand cDNA Synthesis Kit (Fermentas).

\section{PCR and RT-PCR analysis of gene expression}

PCR was performed in a $25 \mu$ l volume containing about 50 ng of genomic DNA of genotype putative transgenic $A$. thaliana lines. Genomic DNA was extracted from fresh young leaves using the cetyltrimethyl ammonium bromide (CTAB) method (Weising et al., 1995). Both Kanamycin-resistant lines and WT plants were tested. The primers used for the amplification of AtchyB, CaMV 35 S promoter and NPT II coding sequences are illustrated in Table 1. PCR amplification cycles were denaturation: $94^{\circ} \mathrm{C}$ for $5 \mathrm{~min}$; denaturation: $94^{\circ} \mathrm{C}$ for $30 \mathrm{sec}$, annealing: $57^{\circ} \mathrm{C}, 54^{\circ} \mathrm{C}$, $58^{\circ} \mathrm{C}$ respectively for AtchyB, CaMV $35 S$ promoter and NPT II for $30 \mathrm{sec}$; primer extention: $72^{\circ} \mathrm{C}$ for $40 \mathrm{sec}, 35$ cycles; and the final extension at $72^{\circ} \mathrm{C}$ for $10 \mathrm{~min}$.

For RT-PCR analysis, $2 \mu$ l of the cDNA product was used in the PCR reaction. Actin (Table 1) was used as the internal control. Actin PCR amplification cycles were denaturation: $94^{\circ} \mathrm{C}$ for $4 \mathrm{~min}$, then $94^{\circ} \mathrm{C}$ for 45 sec; annealing: $60^{\circ} \mathrm{C}$ for $2 \mathrm{~min}$; primer extension: $72^{\circ} \mathrm{C}$ for $3 \mathrm{~min}, 35$ cycles; and the final extension at $72^{\circ} \mathrm{C}$ for $10 \mathrm{~min}$. The PCR and RT-PCR products were analyzed by agarose gel electrophoresis in $0.7 \%$ agarose gels.
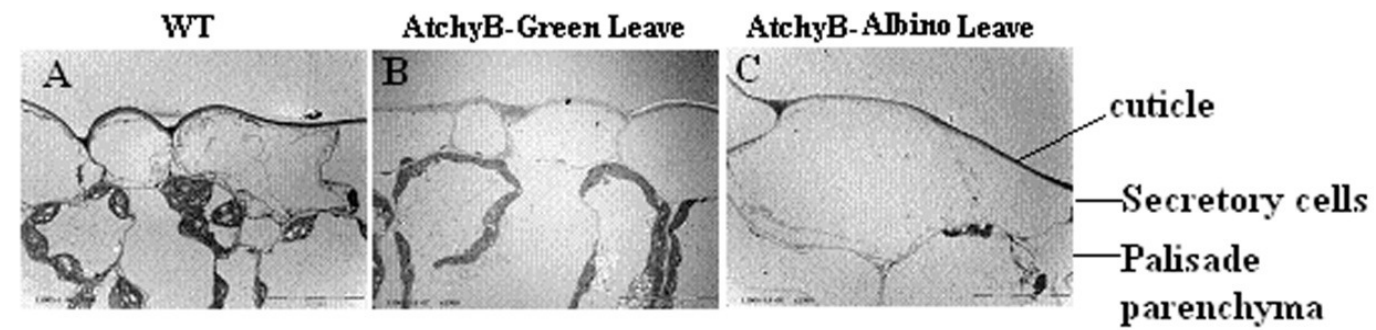

Fig. 4 Cross-section in leaf of WT and transgenic $A$. thaliana showing the upper epidermis ultrastructures. Four weeks old WT and $A t c h y B$ transgenic plants grown in green house were watered daily and once a week with MS nutrient medium. Analysed green and albino plant leaves were sampled on the same plant at the same time. A: upper epidermis in WT plant; B, C: upper epidermis in respectively green and albino leaves of transgenic plant over-expressing the AtchyB gene. All magnifications were X2500. Scale bar $20 \mu \mathrm{m}$.

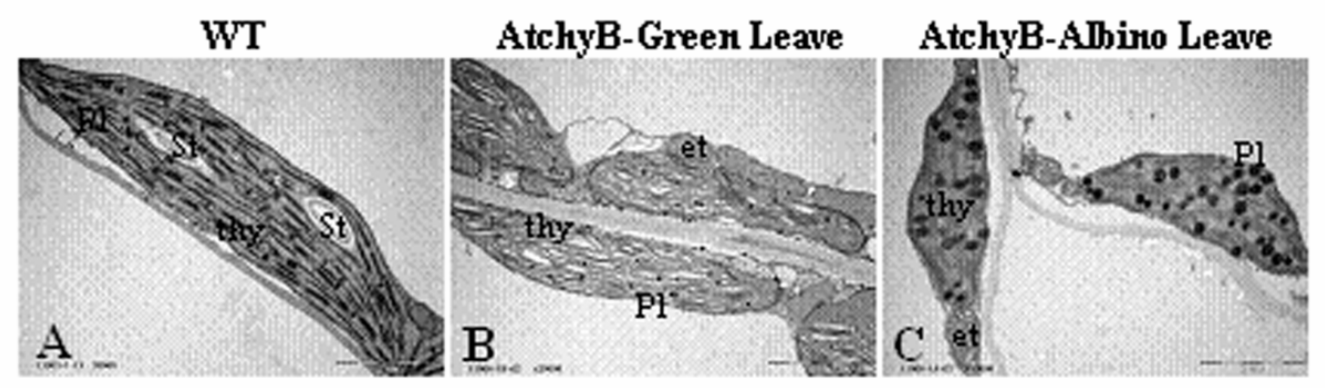

Fig. 5 Cross-section in leaf of WT and transgenic $A$. thaliana showing the chloroplast ultrastructures. Four weeks old WT and AtchyB transgenic plants grown in green house, watered daily and once a week with MS nutrient medium. Analysed green and albino plant leaves were sampled on the same plant at the same time. A: Chloroplast of WT plant showed thylacoids arranged into defined grana, lamellae and starch grains. B: Green leaf chloroplast of $A$. thaliana plant over-expressing the AtchyB gene had thylacoids arranged into define grana and hydrolyzing starch grains. C: Albino leaf of $A$. thaliana plant over-expressing the Atchy $B$ gene. Chloroplasts had thylacoids arranged into define grana. Starch grains are absent. Presence of numerous plastoglobules large in size. Et - etioplast; st - strach; thy - thylacoid; Pl - plastoglobule. All magnifications were X30000. Scale bar $1 \mu \mathrm{m}$. 


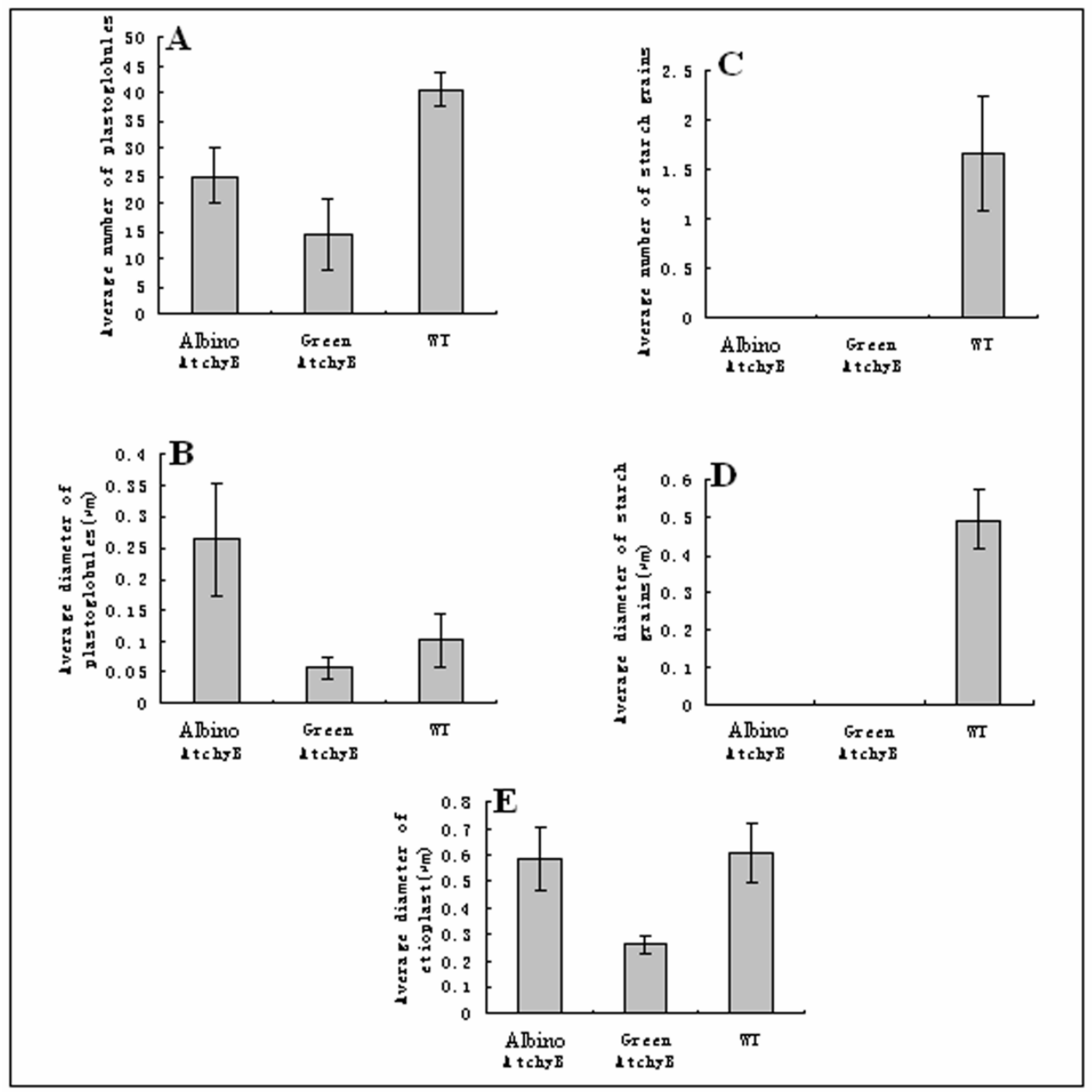

Fig. 6 WT and AtchyB transgenic $A$. thaliana green and albino leaf chloroplast structural components. A: Average number of plastoglobules, B: Average diameter of plastoglobules, C: Average number of starch grains, D: Average diameter of starch grains, E: Average diameter of etioplast. Measurements were taken from one fresh leave of four weeks green house grown WT and AtchyB transgenic A. thaliana. Plants were watered daily and once a week with MS nutrient medium.

\section{Southern-Blot Analysis}

Genomic DNA was isolated from fresh green and albino leaves of putative 4 weeks $\mathrm{T}_{1}$ generation green house grown $A$. thaliana transformants and WT using the CTAB method (Chaudhry et al., 1999). Presence of the transgenes was analysed by PCR and RT-PCR as described above. Southern Blotting was performed as previously described Leila et al. (2012), and based on the protocol of DIG DNA Labeling and Detection Kit (Roche Diagnostics). $20 \mu \mathrm{g}$ genomic DNA was digested with SalI and HindIII, separated electrophoretically on a $1.0 \%(\mathrm{w} / \mathrm{v})$ agarose gel and transferred onto Hybond $\mathrm{N}+$ blotting membrane (Roche Diagnostics) under alkaline conditions, following the manufacturer's instructions. Southern blotting was carried out according to Sambrook and Russell (2001). Probes were produced using DIG labeled a 477 bp AtchyB gene fragment as a probe by PCR DIG Probe Synthesis (Roche Diagnostics).

\section{Transmission Electron Microscopy}

Fully expanded fresh green and albino leaves of 4 weeks T4 plants and WT were acclimated in greenhouse exposed to natural solar radiation with day/night temperature fixed at approximately $28 / 20^{\circ} \mathrm{C}$. Green and albino leaf samples were collected 
on the same plants and from the middle of the blade between the midvein and the margin of the leaf. The upper epidermis, cuticle and chloroplast ultrastructure were analyzed by Transmission Electron Microscope (TEM) according to methods described by (Dylan et al., 2009). Ultrathin sections were observed with a HITACHI-7500 transmission electron microscope (Japan).

\section{Statistical analysis}

All data were subjected to analysis using the SAS software. Experiments were repeated three times and a two-tail $t$-student test was used to compare mean values from green and albino transgenic and WT plants.

\section{Results}

\section{Molecular characterization of the AtchyB gene in transgenic A. thaliana}

At the initial flowering stage, between the 3rd and the 4th week Arabidopsis plants were infected using Agrobacterium tumefaciens mediated transformation with the plasmid pCambia2300-AtchyB carrying the AtchyB and the NPT II genes for selection of kanamycin resistance plants by the floral dipping method. Each screened petri dish of about 100 seeds provided 3\% kanamycin resistance plants. After 10 days, transformants were clearly identifiable as dark green plants with healthy green secondary leaves and roots that extended well into the selective medium (Fig. 1A). Two transgenic lines were obtained. DNA-PCR was AtchyB, NPT $I$ and CaMV $35 S$ promoter positive and RT-PCR analysis confirmed the presence of AtchyB genes in the five transgenic lines. We further determined AtchyB transcript accumulation in transgenic plants. Southern blot revealed L1 line with the highest expression level (two copy numbers). Therefore, the generated transgenic plant AtchyB over-expressing line L1 with the highest AtchyB expression level was used in our experiments (Fig. 2; Fig. 3). T1 was the first kanamycin resistant plant. No obvious morphological difference was observed between the transgenic and WT plants. Instead, primary leaves turned yellowish during the flowering stage and the whole plant developed healthily through the fourth generation (Fig. 1B). T1 plants were used in ultrastructural analysis.

\section{Leaf ultrastructural patterns in green and albino leaves of AtchyB transgenic A. thaliana}

\section{Leaf upper epidermis ultrastructure}

Leaf upper epidermis, chloroplast and cuticle were analyzed in WT and $\mathrm{T}_{1}$ generation of AtchyB over-expressing plants.
Leaf upper epidermis of WT plant, green and albino leaves of transgenic plants were different in configuration. The secretory and the palisade parenchyma cells size were almost similar in WT and AtchyB transgenic green leaves (Fig. 4A, 4B) while the albino leaves presented the largest size of the secretory cells and palisade parenchyma (Fig. 4C). Starch grains disappeared in AtchyB transgenic green leaves (Fig 4B). Very little chloroplast was observed without starch grains in albino leaves when compared with WT and green leaves (Fig. 4C).

\section{Leaf chloroplast ultrastructure}

Electron microscopy in leaf chloroplast of WT, green and albino leaves of $A$. thaliana over-expressing AtchyB revealed mesophyll cells oval in shape. Chloroplast of WT plants showed lamella, starch grains, thylacoids arranged into defined grana and numerous small size plastoglobules could be observed (Fig. 5A). Green leaf of transformed plants had chloroplast with swollen thylacoids arranged into define grana, hydrolyzed starch grains, disperse and very small plastoglobules (Fig 5B). Although albino leaf was not observed with numerous chloroplast, thylacoids were arranged into define grana and increased size of plastoglobules were observed (Fig. 5C).

Starch deposits of usually one to two were found in the chloroplast of WT A. thaliana. No starch grain was observed in AtchyB transgenic green and albino plants leaves (Fig. 6C; 6D). The diameter of etioplast was significantly decreased in green $(0.25 \pm 0.03 \mu \mathrm{m})$ leaf AtchyB transformed plants compare to albino $(0.58 \pm 0.12 \mu \mathrm{m} ; \mathrm{P}=0.024)$ and WT $(0.60 \pm 0.11 \mu \mathrm{m} ; \mathrm{P}=$ 0.027) leaves (Fig.6E). Plastoglobules number was significantly decreased $(\mathrm{P}=0.017)$ in AtchyB transformed plants green $(14.33 \pm 6.42 \mu \mathrm{m})$ leaf and significantly decreased $(\mathrm{P}=0.001)$ in AtchyB transformed plants albino $(25.00 \pm 5.00 \mu \mathrm{m})$ leaf, all compare to WT $(40.66 \pm 3.05 \mu \mathrm{m})$ (Fig. 6A). Although the average diameter of plastoglobules was increased in albino $(0.26 \pm 0.00 \mu \mathrm{m})$ leaves compare to green $(0.05 \pm 0.00 \mu \mathrm{m} ; \quad \mathrm{P}=0.051)$ leaves and WT $(0.10 \pm 0.07 \mu \mathrm{m} ; \mathrm{P}=0.144)$, this difference was not significant (Fig.6B). No starch grains were observed in Atchy $B$ transformed plants green and albino leaf in comparison to WT. This difference was significant [(1.666 $\pm 0.00 ; \mathrm{P}=0.037),(0.494 \pm 0.07 \mu \mathrm{m} ; \mathrm{P}=0.007)$ respectively the average number of starch grains and the average diameter of starch grains] (Fig. 6D, 6C).

\section{Leaf cuticle ultrastructure}

The cuticle thickness of plants over-expressing AtchyB gene was unchanged compare to WT plants. Cellular vesicle compartment at the cell wall side were not filled of epithelial cells in AtchyB transformed plants green and albino leaves compare to WT. The cuticle thickness 

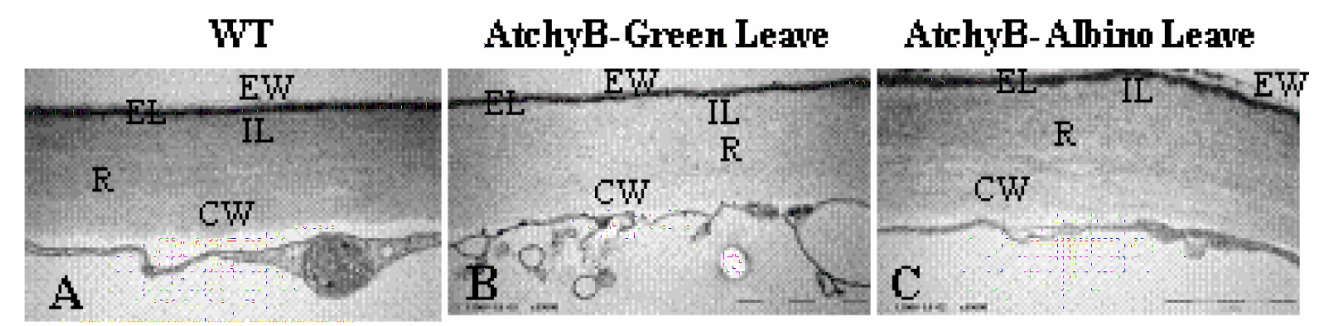

Fig. 7 Cross-section in leaf of WT and transgenic $A$. thaliana showing cuticle ultrastructures. Four weeks old WT and AtchyB transgenic $A$. thaliana plants over-expressing the AtchyB gene grown in green house, watered daily and once a week with MS nutrient medium. Analysed green and albino plant leaves were sampled on the same plant at the same time. A: Cuticle of WT plant. B: Cuticle of green leaf of $A$. thaliana plant over-expressing the AtchyB gene. C: Cuticle of albino leaf of $A$. thaliana plant over-expressing the AtchyB gene. EW - epicuticular waxes; EL - external lamellae; IL - internal lamellae; R - reticulate zone; CW - cell wall. All magnifications were X50000. Scale bar $1 \mu \mathrm{m}$.

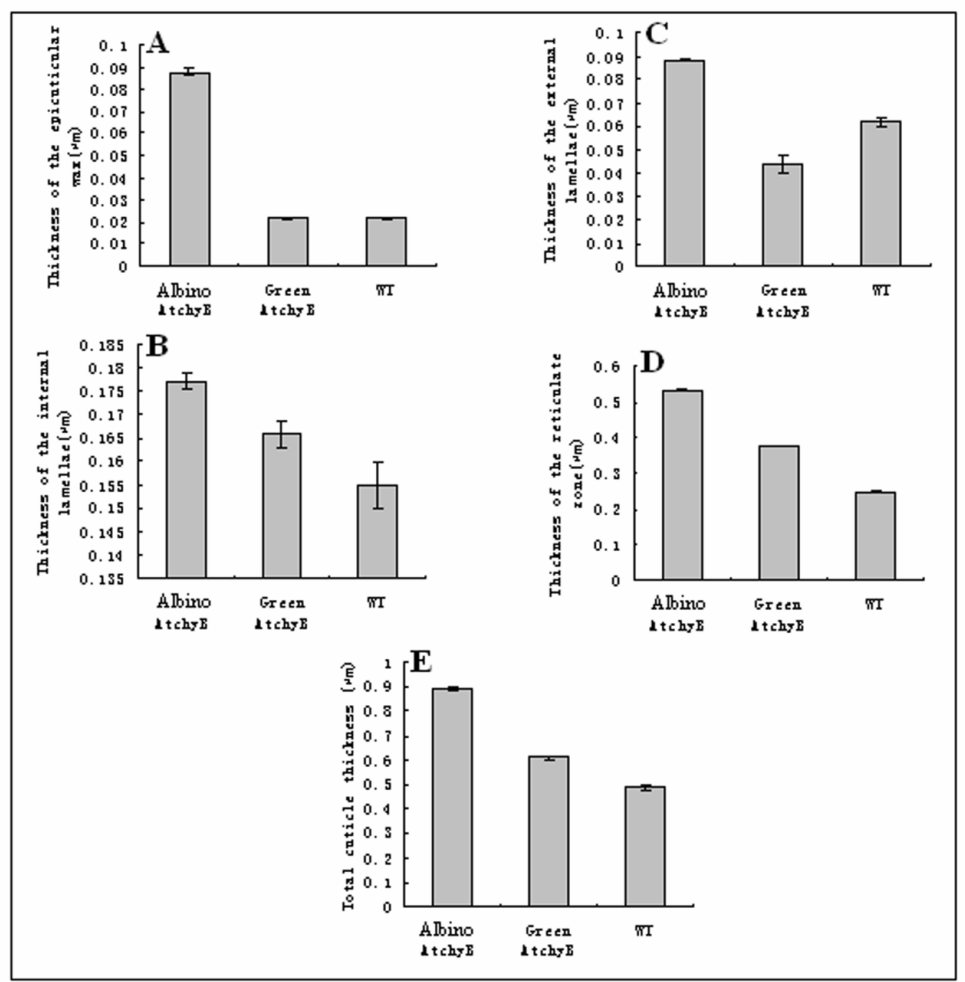

Fig. $8 \mathrm{WT}$ and $A t c h y B$ transgenic $A$. thaliana green and albino leaf cuticle structural components. A: thickness of the epicuticular wax, B: thickness of the internal lamellae, C: thickness of the external lamellae, D: thickness of the reticulate zone, E: total cuticle thickness. Measurements were taken from one fresh leave of four weeks green house grown WT and AtchyB transgenic A. thaliana. Plants were watered daily and once a week with MS nutrient medium.

of albino leaves was increased in comparison to WT and green leaf of AtchyB transformed plants (Fig. 7A; 7B; 7C). The cuticle was smooth in all the samples. The contact between the cuticle and the epidermal cell wall was continuous in albino, green and WT plant leaves.
We also observed a cuticle consisting of a combination of electron lucent and electron dance lamellate zone (Fig. 7A; 7B; 7C). All leaf samples could be observed with the reticulate zone of electron opaque fibrillae coming from the underlying cell wall and punctuated 
with dark granulations. Numerous microvesicles could be seen, bursting out of the plasmalemma.

Quantitative data on the thickness of the cuticle from transgenic plants over-expressing AtchyB (albino and green leaves) and WT leaves is presented in Fig. 8. The thickness of the epicuticular wax was identical in both green leaves of plants over-expressing AtchyB and WT leaves. The epicuticular wax in albino $(0.08 \pm 0.00$ $\mu \mathrm{m})$ leaf was increased in comparison to both green $(0.02 \pm 0.00 \mu \mathrm{m} ; \mathrm{P}=0.0001)$ leaf of AtchyB transgenic and WT $(0.02 \pm 0.00 \mu \mathrm{m} ; \mathrm{P}=0.0004)$ plant leaf (Fig. $8 \mathrm{~A})$. The thickness of the internal lamellae presented a significant decrease between albino $(0.17 \pm 0.00 \mu \mathrm{m})$ and green $(0.16 \pm 0.00 \mu \mathrm{m}),(\mathrm{P}=0.0060)$, albino and $\mathrm{WT}$ $(0.15 \pm 0.00 \mu \mathrm{m}),(\mathrm{P}=0.0081)$, and green and $\mathrm{WT}(\mathrm{P}=$ 0.0108) plant leaves (Fig. 8B). The external lamellae zone consists of electron-dense lamellae alternating with electron-lucent lamellae (internal lamellae). The thickness of the external lamellae was the highest $(0.08 \pm 0.00 \mu \mathrm{m})$ in albino leaves and lowest in AtchyB transformed plants green $(0.04 \pm 0.00 \mu \mathrm{m})$ leaf. These differences were significant between albino and green $(\mathrm{P}=0.0020)$, albino and WT $(0.06 \pm 0.00 \mu \mathrm{m}),(\mathrm{P}=$ $0.0011)$, green and WT $(P=0.0040)$ plant leaves (Fig. 8C). The thickness of the reticulate zone and that of the total cuticle thickness had the same variation as that of the internal lamellae. Differences were significant between albino and green $\left(\mathrm{P}=4 \times 10^{-5} ; \mathrm{P}=8 \times 10^{-5}\right)$, albino and WT $\left(\mathrm{P}=3 \times 10^{-5} ; \mathrm{P}=2 \times 10^{-5}\right)$, and green and WT $\left(\mathrm{P}=4 \times 10^{-5} ; \mathrm{P}=5 \times 10^{-5}\right)$ plant leaves of the reticulate zone and the total cuticle thickness respectively (Fig. 8D; 8E).

\section{Discussion}

AtchyB gene was previously investigated to mediate high light stress in Eustoma grandiflorum Shinn (Weidang et al., 2012), as well as high light and high temperature stresses in A. thaliana (Davison et al., 2002). This enzyme is involved in the conversion of $\beta$-carotene into zeaxanthin and plays an important role in the pathway of carotenoid biosynthesis. In this study we assessed the input of AtchyB gene to the T1 generation of $A$. thaliana albino and green leaf ultrastructural response. Expression of AtchyB cDNA govern by CaMV $35 S$ promoter showed ultrastructural changes in transgenic albino and green Arabidopsis plant leaves compare to WT plant leaf. The progressive yellow coloration of the AtchyB transformed plants was due to hight carotenoids production and obviously the presence of the AtchyB gene.

Differential features were observed, condensed and orthodox configurational states in green and albino leaves of $A$. thaliana over-expressing the Atchy $B$ gene comparable with those exhibited in WT. The insertion of AtchyB gene to A. thaliana activates the dilatation of the palisade parenchyma in green leaf and the dilatation of both the palisade parenchyma and the secretory cells in albino leaf. The AtchyB gene also induced a reduction in the number of chloroplast and starch grains in green leaf, while both elements were nearly absent in albino leaves. Indicating that, AtchyB gene induces albinism in $A$. thaliana primary leaves, obviously reducing the ability of these leaves to synthesize chlorophyll. Although only very few chloroplast were present in albino leaf, numerous large size plastoglobules could be observed. The AtchyB gene induction of albinism may induce water deficit leading to an increase of cuticle thickness (Dylan et al., 2009). Albino leaves of $A$. thaliana over-expressing the AtchyB gene may not support plant metabolism due to the breakdown in the synthesis of starch and chlorophyll. Green pigmentation is important for leaf energy absorption and photosynthesis (Carter and Stein, 1996). The same phenomenon was previously observed in maltose excess 1 mutant (mex1) in $A$. thaliana (Michaela et al. 2009). zebra7(zb7) mutant maize were found by Xiao-Min et al. (2012) with decreased levels of chlorophylls and delayed chloroplast development in their yellow bands.

We could conclude that, AtchyB gene over-expression in $A$. thaliana induces albinism that may cause yellow leaves water deficit, chloroplast dysfunction and retrograde signaling associated with starch and chloroplast degradation. Additionally, Atchy $B$ gene over-expression stimulates in A. thaliana albinism leaves the synthesis of numerous large size plastoglobules and a dilatation of both the palisade parenchyma and the secretory cells. These demonstrated that AtchyB gene over-expression in $A$. thaliana induces albinism that affects albino leaves ultrastructure with retrograde signaling, defects in chlorophyll biosynthesis and chloroplast dysfunction. The AtchyB gene induces reduction in Leaf chlorophyll pigmentation that may affect energy absorption and photosynthesis, especially in albino leaves.

\section{Acknowledgments}

This work was supported by the China Postdoctoral Science Foundation under grant (ZF2012041) and the National Natural Science Foundation under grant (31271793, 31271419).

\section{References}

Almeida, L. S., N. Maia, A. R. Ortega, and A. C. Angelo. 2005. Crescimento de mudas de Jacaranda puberula Cham. Em viveiro submetidas a diferentes niveis de sombreamento. Ciência Florestal 15(3): 323-329.

Abadie, J. C., U. Puttsepp, G. Gebauer, A. Faccio, P. Bonfante, and M. A. Selosse. 2006. Cephalanthera longifolia (Neottieae, Orchidaceae) is 
mixotrophic: a comparative study between green and nonphotosynthetic individuals. Canadian Journal of Botany 84: 1462-1477. doi:10.1139/b06-101

Abdelkader, A.F., H. Aronsson, K. Solymosi, B. Böddi, and C. Sundqvist. 2007. Highsalt stress induces swollen prothylakoids in dark-grown wheat and alters both prolamellar body transformation and reformation after irradiation. Journal of Experimental Botany 58: 2553-2564. doi:10.1093/jxb/erm085

Carter, J. S. 1996. Photosynthesis. University of Cincinnati.

Claire, B., K. Felix ${ }_{2}$ and J. van.W. Klaas. 2007. Plastoglobules: versatile lipoprotein particles in plastids. Trends in Plant Science 12(6): 260-6. doi:10.1016/j.tplants.2007.04.003

Chaudhry, B., Y. Afshan, T. Husnain, and S. Riazuddin. 1999. Mini-scale genomic dna extraction from cotton. Plant Molecular Biology Reporter 17: 1-7. doi:10.1023/A:1007629715971

Davison, P. A., C. N. Hunter, and P. Horton. 2002. Overexpression of beta-carotene hydroxylase enhances stress tolerance in Arabidopsis. Nature 418(6894): 203-206. doi:10.1038/nature00861

Dylan, K., B. B. Kosma, L. B. Ame, P. P. Eugene, L. Shiyou, J. Jerome, and A. J. Matthew. 2009. The impact of water deficiency on leaf cuticle lipids of Arabidopsis. Plant Physiology 151: 1918-1929. doi:10.1104/pp.109.141911

Helen, B-M., and G. Eric. 2014. Carotenoid metabolism is induced in rice bran during very high temperature stress. Journal of the Science of Food and Agriculture doi:10.1002/jsfa.6496.

Leila, P., A. H. Ali, J. K. Maryam, K. Ahmad, and T. Zahra. 2012. Agrobacterium-media tedtransformation of chitinase gene in Rosa damascene cv. Ghamsar. Annals of Biological Research 3(6): 2843-2850.

Maya, K., J. C. Heather, S. Ian, and H. M. S. Kadambot. 2009. Albinism in plants: A major bottleneck in wide hybridization, androgenesis and doubled haploid culture. Critical Reviews in Plant Sciences 28(6): 393-409. doi:10.1080/07352680903133252.

Michaela. S., E. Simona, M. Tabea, M. Gaelle, H. Stefan, and C. Z. Samuel. 2009. Blocking the metabolism of starch breakdown products in Arabidopsis leaves triggers chloroplast degradation. Molecular Plant 2(6): 1233-1246. doi:10.1093/mp/ssp093

Qi, Y. C., S. M. Zhang, L. P. Wang, M. D. Wang, and H. Zhang. 2004. Overexpression of GST accelerates the growth of transgenic Arabidopsis under the salt stress. Journal of Plant Physiology and molecular Biology. 30. 517-552.
Sambrook, J., and D. W. Russell. 2001. Molecular cloning: a laboratoryManual. 3nd ed. NewYork: Cold Spring Harbor Laboratory Press.

Tchouopou, L. J., J. Jing, W. Gang, Z. Qing, F. G. Chun, and L. Y. Hai. 2013. AtchyB transgenic Arabidopsis thaliana; Leaf physiological and ultrastructural changes to salinity and the effect of exogenous $\mathrm{H} 2 \mathrm{O} 2, \mathrm{LiCl}, \mathrm{NaCl}$ to seed germination and root elongation. Bothalia Journal. 43(11): 20-47.

Weidang, W., J. Jing, W. Gang, Z. Qing, J. Chao, G. Chunfeng, and L. J. Tchouopou. 2012. Overexpression of AtchyB in Eustoma grandiflorum Shinn enhances its tolerance to high-light via zeaxanthin accumulation. Plant Molecular Biology Reporter 30(6): 1433-1443. doi:10.1007/s11105-012-0460-4

Weising, K., H. Nybom, K. Wolff, and W. Meyer. 1995. DNA fingerprinting in plants and fungi. CRC Press, Boca Raton, Fla.

Yua, Q-B., Q. Ma, M-M. Kong, T-T. Zhao, X-L. Zhang, Q. Zhou, C. Huang, K. Chong, and Z-N.Yang. 2014. AtECB1/MRL7, a thioredoxin-like fold protein with disulfide reductase activity, regulates chloroplast gene expression and chloroplast biogenesis in Arabidopsis thaliana. Molecular Plant 7(1) p206. doi:10.1093/mp/sst092

Wang, M., G. Wang, J. Ji, and J-H. Wang. 2009. The effect of $p d s$ gene silencing on chloroplast pigment composition, thylakoid membrane structure and photosynthesis efficiency in tobacco plants. Plant Science 177(3): 222-226. doi:10.1016/j.plantsci.2009.04.006

Xiao-Min, L., H. Xiao-Jiao, Z. Yuan-Zeng, S. Wei-Bin, Z. Mei, C. Zong-Liang, C. Wei, D. Yong-Bin, W. Zhen-Hua, and L. Jin-Sheng. 2012. Map-Based Cloning of zb7 Encoding an IPP and DMAPP Synthase in the MEP Pathway of Maize. Molecular plant 5(5): 1100-1112. doi:10.1093/mp/sss038

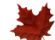

